\title{
Periodic Conservative Solutions for a Modified Two-Component Camassa-Holm System with Peakons
}

\author{
Yujuan Wang and Yongduan Song \\ School of Automation, Chongqing University, Chongqing 400044, China \\ Correspondence should be addressed to Yongduan Song; ydsong@cqu.edu.cn \\ Received 11 August 2013; Accepted 24 October 2013 \\ Academic Editor: Sining Zheng
}

Copyright (c) 2013 Y. Wang and Y. Song. This is an open access article distributed under the Creative Commons Attribution License, which permits unrestricted use, distribution, and reproduction in any medium, provided the original work is properly cited.

This paper investigates the periodically weak conservative solutions for a modified periodic two-component Camassa-Holm system arisen from shallow water waves moving. The underlying approach is based on a system reformulation by introducing a skillfully defined characteristic and a set of newly defined variables. Such treatment makes it possible to overcome the difficulties inherent in the multicomponent system, leading to the establishment of the periodic conservative solutions for the original system.

\section{Introduction}

We consider in this paper a modified periodic twocomponent Camassa-Holm system with peakons [1], namely,

$$
\begin{aligned}
m_{t}+2 m u_{x}+m_{x} u+(m v)_{x}+n v_{x} & =0, \\
t & >0, x \in R, \\
n_{t}+2 n v_{x}+n_{x} v+(n u)_{x}+m u_{x} & =0, \\
t & >0, x \in R,
\end{aligned}
$$

with the initial and periodic conditions

$$
\begin{aligned}
& u(0, x)=u_{0}(x), \quad x \in R, \\
& v(0, x)=v_{0}(x), \quad x \in R, \\
& u(t, x+1)=u(x), \quad t>0, x \in R, \\
& v(t, x+1)=v(x), \quad t>0, \quad x \in R,
\end{aligned}
$$

where $m=u-u_{x x}, n=v-v_{x x}$ are periodic on the $x$ variable. System (1) is a modified version of the coupled twocomponent Camassa-Holm system in [2],

$$
\begin{gathered}
m_{t}=2 m u_{x}+m_{x} u+(m v)_{x}+n v_{x}, \quad t>0, x \in R, \\
n_{t}=2 n v_{x}+n_{x} v+(n u)_{x}+m u_{x}, \quad t>0, x \in R,
\end{gathered}
$$

which has been established by $\mathrm{Fu}$ and $\mathrm{Qu}$ to allow for peakon solitons in the form of a superposition of multipeakons. By parameterizing $\widetilde{t}=-t$ for system (3), it then takes the form of (1), which can be rewritten as a Hamiltonian system,

$$
\frac{\partial}{\partial t}\left(\begin{array}{c}
m \\
n
\end{array}\right)=-\left(\begin{array}{ll}
\partial m+m \partial & \partial m+n \partial \\
\partial n+m \partial & \partial n+n \partial
\end{array}\right)\left(\begin{array}{l}
\frac{\delta H}{\delta m}=u \\
\frac{\delta H}{\delta n}=v
\end{array}\right)
$$

with the Hamiltonian $H=(1 / 2) \int(m G * m+n G * n) d x$, where $G * m=u, G * n=v$, and $G=(1 / 2) e^{-|x|}$. Furthermore, it has the following conserved quantities:

$$
\int_{S} u d x, \int_{S} v d x, \int_{S} m d x, \int_{S} n d x, \int_{S}\left(u^{2}+u_{x}^{2}+v^{2}+v_{x}^{2}\right) d x .
$$

$$
\begin{aligned}
& \text { A remarkable fact for the two types of coupled two- } \\
& \text { component Camassa-Holm system is that they have an }
\end{aligned}
$$


infinite number of peakons, which take the following form in the periodic case that

$$
\begin{aligned}
& u(t, x) \\
& =\frac{1}{\sinh (1 / 2)} \sum_{i=1}^{n}\left(p_{i}(t) \cosh \left(x-q_{i}(t)-\left[x-q_{i}(t)\right]-\frac{1}{2}\right)\right), \\
& v(t, x) \\
& =\frac{1}{\sinh (1 / 2)} \sum_{i=1}^{n}\left(r_{i}(t) \cosh \left(x-q_{i}(t)-\left[x-q_{i}(t)\right]-\frac{1}{2}\right)\right),
\end{aligned}
$$

where $\left(p_{i}(t), r_{i}(t), q_{i}(t)\right)$ satisfy the explicit system of ordinary differential equations

$$
\begin{aligned}
& \dot{p}_{i}=\sum_{j=1, i \neq j}^{n}\left(p_{i} p_{j}+r_{i} r_{j}\right) \operatorname{sgn}\left(q_{j}-q_{i}\right) e^{-\left|q_{i}-q_{j}\right|}, \\
& \dot{r}_{i}=\sum_{j=1, i \neq j}^{n}\left(p_{i} p_{j}+r_{i} r_{j}\right) \operatorname{sgn}\left(q_{j}-q_{i}\right) e^{-\left|q_{i}-q_{j}\right|}, \\
& \dot{q}_{i}=-\sum_{j=1}^{n}\left(p_{j}+r_{j}\right) e^{-\left|q_{i}-q_{j}\right|} .
\end{aligned}
$$

In particular, when $u=0$ (or $v=0$ ), system (1) has the same peakon solitons as the Camassa-Holm $(\mathrm{CH})$ equation.

Moreover, when $u=v$, system (1) is reduced to the scalar Camassa-Holm equation,

$$
m_{t}+4 m u_{x}+2 m_{x} u=0 .
$$

The $\mathrm{CH}$ equation which models the unidirectional propagation of shallow water waves over a flat bottom has attracted considerable attention because it has a bi-Hamiltonian structure [3] and is completely integrable [4, 5]. Another interesting property of the $\mathrm{CH}$ equation is that the solutions experience wave breaking $[4,6,7]$. The presence of breaking waves means that the solution remains bounded while its slope becomes unbounded in finite time [7, 8]. After wave breaking the solutions of the $\mathrm{CH}$ equation can be continued uniquely as either global conservative [9-12] or global dissipative solutions [13].

The important issue to address is the Cauchy problem for system (1) in the periodic case on $S$. It has been shown that system (1) is locally well posed with periodic initial value and there exist global strong solutions which blow up in finite time for certain class of initial data [1]. Moreover, an existence result for a class of lower regularity of solutions [1] and the global attractor with the periodic boundary condition [14] were also given.

It should be noted that extending the solutions beyond wave breaking imposes significant challenge, as reflected in the case of multipeakons given by (6). Peakons interact in a way similar to that of solitons of the $\mathrm{CH}$ equation, and wave breaking may appear when at least two of the $q_{i}$ coincide. However, the solutions (6) are not smooth even with continuous functions $\left(p_{i}(t), r_{i}(t), q_{i}(t)\right)$. One possible way to interpret (6) as a weak solution of system (1) is to rewrite (6) as

$$
\begin{aligned}
u_{t}+(u+v) u_{x}+\left(1-\partial_{x}^{2}\right)^{-1} & \\
\times\left[u v_{x}+\left(u^{2}+\frac{1}{2} u_{x}^{2}+u_{x} v_{x}+\frac{1}{2} v^{2}-\frac{1}{2} v_{x}^{2}\right)_{x}\right]=0, & t>0, x \in R, \\
v_{t}+(u+v) v_{x}+\left(1-\partial_{x}^{2}\right)^{-1} & \quad t>0, \quad x \in R .
\end{aligned}
$$

In our recent work $[15,16]$, we investigate the continuity of the solutions for system (1) beyond collision time on the full line, leading to uniquely to either global conservative solutions where the energy is conserved for almost all times or global dissipative solutions where energy loss occurs only through wave breaking.

Motivated by our recent work [15-17], we study in this paper the continuation of the solutions for system (1) beyond wave breaking in the periodic case and present a novel approach from a Lagrangian point of view. The approach we applied here is mainly based on the reformulation by introducing a newly defined characteristic and a set of variables, where the associated energy is introduced as an additional variable to obtain a well-posed initial-value problem. The argument is inspired mainly by [11] in the study of the periodic conservative solution of the $\mathrm{CH}$ equation. However, one problematic issue is that we here deal with a coupled system where the mutual effect between two components makes the analysis more complicated than a single one. The newly introduced characteristic and the skillfully defined variables are crucial to overcome the above-mentioned problems. By overcoming the problems, we obtain the global periodic conservative solutions of (1).

The outline of this paper is as follows. The original system is transformed into a Lagrangian equivalent semilinear system by introducing a set of newly defined variables in Section 2 and the global solutions of the equivalent system are obtained in Section 3. Section 4 is devoted to establishing the periodic conservative solutions for the original system, which construct a global continuous semigroup. The paper is just closed in Section 5.

1.1. Notation. Throughout this paper, we identify all spaces of periodic functions with function spaces over the unit circle $S$ in $R^{2}$; that is, $S=R / Z$. We denote by $*$ the spatial convolution.

\section{The Original System and the Lagrangian Equivalent System}

2.1. Part A: The Original System. Let us introduce an operator $\Lambda=\left(1-\partial_{x}^{2}\right)^{-1}$, associated with its Green function 
$G(x)=(\cosh (x-[x]-1 / 2)) /(2 \sinh (1 / 2))$, where $[x]$ stands for the integer part of $x$, such that

$$
\begin{array}{r}
\Lambda f(x)=G * f(x) \\
=\frac{1}{2} \int_{0}^{1} \frac{\cosh \left(x-x^{\prime}-\left[x-x^{\prime}\right]-1 / 2\right)}{\sinh (1 / 2)} f\left(x^{\prime}\right) d x^{\prime}, \\
f \in L^{2}(S) .
\end{array}
$$

Thus we can rewrite (1) as a form of a quasilinear evolution equation,

$$
\begin{array}{r}
u_{t}+(u+v) u_{x}+G *\left(u v_{x}\right) \\
+\partial_{x} G *\left(u^{2}+\frac{1}{2} u_{x}^{2}+u_{x} v_{x}+\frac{1}{2} v^{2}-\frac{1}{2} v_{x}^{2}\right)=0, \\
t>0, x \in R, \\
v_{t}+(u+v) v_{x}+G *\left(u_{x} v\right) \\
+\partial_{x} G *\left(v^{2}+\frac{1}{2} v_{x}^{2}+u_{x} v_{x}+\frac{1}{2} u^{2}-\frac{1}{2} u_{x}^{2}\right)=0, \\
t>0, x \in R .
\end{array}
$$

If we define $A(t, x), B(t, x), C(t, x)$, and $D(t, x)$ as follows:

$$
\begin{aligned}
A(t, x) & =G *\left(u v_{x}\right) \\
& =\frac{1}{2} \int_{0}^{1} \frac{\cosh \left(x-x^{\prime}-\left[x-x^{\prime}\right]-1 / 2\right)}{\sinh (1 / 2)}\left(u v_{x}\right)\left(t, x^{\prime}\right) d x^{\prime} \\
B(t, x) & =G *\left(u^{2}+\frac{1}{2} u_{x}^{2}+u_{x} v_{x}+\frac{1}{2} v^{2}-\frac{1}{2} v_{x}^{2}\right) \\
& =\frac{1}{2} \int_{0}^{1} \frac{\cosh \left(x-x^{\prime}-\left[x-x^{\prime}\right]-1 / 2\right)}{\sinh (1 / 2)} \\
C(t, x)= & G *\left(v u_{x}\right) \\
= & \frac{1}{2} \int_{0}^{1} \frac{\cosh \left(x-x^{\prime}-\left[x-x^{\prime}\right]-1 / 2\right)}{\sinh (1 / 2)}\left(v u_{x}\right)\left(t, x^{\prime}\right) d x^{\prime} \\
D(t, x)= & G *\left(v_{x} v_{x}+\frac{1}{2} v^{2}-\frac{1}{2} v_{x}^{2}\right)\left(t, x^{\prime}\right) d x^{\prime} \\
& =\frac{1}{2} \int_{0}^{1} \frac{\cosh \left(x-x_{x}^{2}+u_{x} v_{x}+\frac{1}{2} u^{2}-\frac{1}{2} u_{x}^{2}\right)}{\sinh (1 / 2)} \\
& \times\left(v^{2}+\frac{1}{2} v_{x}^{2}+u_{x} v_{x}+\frac{1}{2} u^{2}-\frac{1}{2} u_{x}^{2}\right)\left(t, x^{\prime}\right) d x^{\prime},
\end{aligned}
$$

then (1) can be rewritten as

$$
\begin{aligned}
& u_{t}+(u+v) u_{x}+A+B_{x}=0, \quad t>0, x \in R, \\
& v_{t}+(u+v) v_{x}+C+D_{x}=0, \quad t>0, x \in R .
\end{aligned}
$$

For smooth solutions, we have that the total energy

$$
E(t)=\int_{S}\left(u^{2}+u_{x}^{2}+v^{2}+v_{x}^{2}\right)(t, x) d x
$$

is constant in time. Indeed, differentiating the two equations in (13), respectively, with respect to $x$ and using the identity $\partial_{x}^{2} G * f=G * f-f$, we have

$$
\begin{aligned}
u_{x t}+ & u_{x}^{2}+u_{x} v_{x}+u u_{x x}+v u_{x x}+A_{x} \\
& +B-\left(u^{2}+\frac{u_{x}^{2}}{2}+u_{x} v_{x}+\frac{v^{2}}{2}-\frac{v_{x}^{2}}{2}\right)=0, \\
v_{x t}+ & v_{x}^{2}+u_{x} v_{x}+v v_{x x}+u v_{x x}+C_{x} \\
& +D-\left(v^{2}+\frac{v_{x}^{2}}{2}+u_{x} v_{x}+\frac{u^{2}}{2}-\frac{u_{x}^{2}}{2}\right)=0 .
\end{aligned}
$$

Multiplying the first equation in (13) by $u$ and the second by $v$ and multiplying the first equation in (15) by $u_{x}$ and the second by $v_{x}$, we obtain the following four equalities:

$$
\begin{gathered}
\left(\frac{u^{2}}{2}\right)_{t}+\left(\frac{u^{3}}{3}\right)_{x}+u v u_{x}+u A+u B_{x}=0, \\
\left(\frac{v^{2}}{2}\right)_{t}+\left(\frac{v^{3}}{3}\right)_{x}+u v v_{x}+v C+v D_{x}=0, \\
\left(\frac{u_{x}^{2}}{2}\right)_{t}+\left(\frac{u u_{x}^{2}}{2}-\frac{u^{3}}{3}\right)_{x}+v u_{x} u_{x x}+u^{2} v_{x} \\
-u A+u_{x} B-\frac{v^{2} u_{x}}{2}+\frac{v_{x}^{2} u_{x}}{2}=0, \\
\left(\frac{v_{x}^{2}}{2}\right)_{t}+\left(\frac{v v_{x}^{2}}{2}-\frac{v^{3}}{3}\right)_{x}+u v_{x} v_{x x}+v^{2} u_{x} \\
-v C+v_{x} D-\frac{u^{2} v_{x}}{2}+\frac{u_{x}^{2} v_{x}}{2}=0 .
\end{gathered}
$$

Using the four identities in (16a), (16b), (16c), and (16d), we can get that

$$
\frac{d}{d t} E(t)=\frac{d}{d t} \int_{S}\left(u^{2}+u_{x}^{2}+v^{2}+v_{x}^{2}\right)(t, x) d x=0 .
$$

Thus (13) possesses the $H_{\mathrm{per}}^{1}$-norm conservation law defined as

$$
\begin{aligned}
\|z\|_{H_{\mathrm{per}}^{1}} & =\|u\|_{H_{\mathrm{per}}^{1}}+\|v\|_{H_{\mathrm{per}}^{1}} \\
& =\left(\int_{[0,1]}\left[u^{2}+u_{x}^{2}+v^{2}+v_{x}^{2}\right] d x\right)^{1 / 2},
\end{aligned}
$$

where $z(t, x)=(u, v)(t, x)$ denotes the solution of system (13). Since $z \in H_{\mathrm{per}}^{1}$, Young's inequality ensures $A, B, C, D \in H_{\mathrm{per}}^{1}$. 
2.2. Part B: The Lagrangian Equivalent System. We reformulate system (13) as follows. We first introduce the corresponding characteristic $y: R \rightarrow W_{1}, t \rightarrow y(t, \cdot)$ as the solution of

$$
y_{t}(t, \xi)=(u+v)(t, y(t, \xi))
$$

where the space $W_{1}$ is defined by $W_{1}=\left\{f \in H_{\text {loc }}^{1}(R) \mid f(\xi+\right.$ $1)=f(\xi)+1$ for all $\xi \in R\}$. We define the cumulative energy distribution $H$ as

$$
H(t, \xi)=\int_{y(t, 0)}^{y(t, \xi)}\left(u^{2}+u_{x}^{2}+v^{2}+v_{x}^{2}\right)(t, x) d x
$$

It is not hard to check that

$$
\begin{aligned}
\left(u^{2}+\right. & \left.u_{x}^{2}+v^{2}+v_{x}^{2}\right)_{t}+\left((u+v)\left(u^{2}+u_{x}^{2}+v^{2}+v_{x}^{2}\right)\right)_{x} \\
& =\left(u^{3}-2 u B+v^{3}-2 v D\right)_{x} .
\end{aligned}
$$

Then it follows from (19) and (21) that

$$
\frac{d H}{d t}=\left[\left(u^{3}-2 u B+v^{3}-2 v D\right)(t, y(t, \xi))\right]_{0}^{\xi}
$$

Using the fact that $y \in W_{1}$ and the periodicity of $u$ and $v$, it follows from (20) and (22) that

$$
\begin{aligned}
& H(t, \xi+1)-H(t, \xi) \\
& \quad=H(t, 1)-H(t, 0)=H(0,1)-H(0,0)
\end{aligned}
$$

for all $\xi \in R$. Let us introduce the vector space $W$ given by

$$
\begin{gathered}
W=\left\{f \in H_{\mathrm{loc}}^{1}(R) \mid f(\xi+1)=f(\xi)+\alpha\right. \\
\text { for some } \alpha \in R \text {, all } \xi \in R\}
\end{gathered}
$$

endowed with the norm $\|f\|_{W}=\|f\|_{H^{1}([0,1])}$, which is a Banach space. Then we have that $H \in W$.

Throughout the following, we use the notation

$$
\begin{aligned}
U(t, \xi) & =u(t, y(t, \xi)), & V(t, \xi) & =v(t, y(t, \xi)), \\
U_{x}(t, \xi) & =u_{x}(t, y(t, \xi)), & V_{x}(t, \xi) & =v_{x}(t, y(t, \xi)) .
\end{aligned}
$$

After the change of variables $x=y(t, \xi)$ and $x^{\prime}=y\left(t, \xi^{\prime}\right)$, we obtain the following expressions for $A, A_{x}, B, B_{x}, C, C_{x}, D$, and $D_{x}$, namely,

$$
\begin{aligned}
A(\xi)=\frac{1}{2} \int_{0}^{1} & \frac{\cosh \left(y(\xi)-y\left(\xi^{\prime}\right)-\left[y(\xi)-y\left(\xi^{\prime}\right)\right]-1 / 2\right)}{\sinh (1 / 2)} \\
& \times\left[\left(U V_{x}\right) y_{\xi}\right]\left(\xi^{\prime}\right) d \xi^{\prime}
\end{aligned}
$$$$
A_{x}(\xi)=\frac{1}{2} \int_{0}^{1} \frac{\sinh \left(y(\xi)-y\left(\xi^{\prime}\right)-\left[y(\xi)-y\left(\xi^{\prime}\right)\right]-1 / 2\right)}{\sinh (1 / 2)}
$$$$
\times\left[\left(U V_{x}\right) y_{\xi}\right]\left(\xi^{\prime}\right) d \xi^{\prime}
$$

$B(\xi)=\frac{1}{4} \int_{0}^{1} \frac{\cosh \left(y(\xi)-y\left(\xi^{\prime}\right)-\left[y(\xi)-y\left(\xi^{\prime}\right)\right]-1 / 2\right)}{\sinh (1 / 2)}$

$$
\begin{gathered}
\cdot\left[H_{\xi}+\left(U^{2}+2 U_{x} V_{x}-V_{x}^{2}\right) y_{\xi}\right]\left(\xi^{\prime}\right) d \xi^{\prime}, \\
B_{x}(\xi)=\frac{1}{4} \int_{0}^{1} \frac{\sinh \left(y(\xi)-y\left(\xi^{\prime}\right)-\left[y(\xi)-y\left(\xi^{\prime}\right)\right]-1 / 2\right)}{\sinh (1 / 2)} \\
\cdot\left[H_{\xi}+\left(U^{2}+2 U_{x} V_{x}-V_{x}^{2}\right) y_{\xi}\right]\left(\xi^{\prime}\right) d \xi^{\prime}, \\
C(\xi)=\frac{1}{2} \int_{0}^{1} \frac{\cosh \left(y(\xi)-y\left(\xi^{\prime}\right)-\left[y(\xi)-y\left(\xi^{\prime}\right)\right]-1 / 2\right)}{\sinh (1 / 2)} \\
\quad \times\left[\left(V U_{x}\right) y_{\xi}\right]\left(\xi^{\prime}\right) d \xi^{\prime},
\end{gathered}
$$$$
C_{x}(\xi)=\frac{1}{2} \int_{0}^{1} \frac{\sinh \left(y(\xi)-y\left(\xi^{\prime}\right)-\left[y(\xi)-y\left(\xi^{\prime}\right)\right]-1 / 2\right)}{\sinh (1 / 2)}
$$$$
\times\left[\left(V U_{x}\right) y_{\xi}\right]\left(\xi^{\prime}\right) d \xi^{\prime}
$$$$
D(\xi)=\frac{1}{4} \int_{0}^{1} \frac{\cosh \left(y(\xi)-y\left(\xi^{\prime}\right)-\left[y(\xi)-y\left(\xi^{\prime}\right)\right]-1 / 2\right)}{\sinh (1 / 2)}
$$$$
\cdot\left[H_{\xi}+\left(V^{2}+2 U_{x} V_{x}-U_{x}^{2}\right) y_{\xi}\right]\left(\xi^{\prime}\right) d \xi^{\prime}
$$

$D_{x}(\xi)=\frac{1}{4} \int_{0}^{1} \frac{\sinh \left(y(\xi)-y\left(\xi^{\prime}\right)-\left[y(\xi)-y\left(\xi^{\prime}\right)\right]-1 / 2\right)}{\sinh (1 / 2)}$

$$
\cdot\left[H_{\xi}+\left(V^{2}+2 U_{x} V_{x}-U_{x}^{2}\right) y_{\xi}\right]\left(\xi^{\prime}\right) d \xi^{\prime},
$$

where the variable $t$ is omitted for simplification and in the above we used the fact that $H_{\xi}=\left(u^{2}+u_{x}^{2}+v^{2}+v_{x}^{2}\right) \circ y y_{\xi}$ and took for granted that $y$ is an increasing function for any fixed time $t$ (the validity will be proved later). In view 
of the periodicity of $H_{\xi}, U$, and $V$, these expressions can be rewritten as

$$
\begin{aligned}
& A(\xi)=\frac{1}{e-1} \int_{0}^{1} \cosh \left(y(\xi)-y\left(\xi^{\prime}\right)\right)\left[\left(U V_{x}\right) y_{\xi}\right]\left(\xi^{\prime}\right) d \xi^{\prime} \\
& +\frac{1}{2} \int_{0}^{1} e^{-\left|y(\xi)-y\left(\xi^{\prime}\right)\right|}\left[\left(U V_{x}\right) y_{\xi}\right]\left(\xi^{\prime}\right) d \xi^{\prime}, \\
& A_{x}(\xi)=\frac{1}{e-1} \int_{0}^{1} \sinh \left(y(\xi)-y\left(\xi^{\prime}\right)\right)\left[\left(U V_{x}\right) y_{\xi}\right]\left(\xi^{\prime}\right) d \xi^{\prime} \\
& -\frac{1}{2} \int_{0}^{1} \operatorname{sgn}\left(\xi-\xi^{\prime}\right) e^{-\left|y(\xi)-y\left(\xi^{\prime}\right)\right|} \\
& \times\left[\left(U V_{x}\right) y_{\xi}\right]\left(\xi^{\prime}\right) d \xi^{\prime}, \\
& B(\xi)=\frac{1}{2(e-1)} \\
& \times \int_{0}^{1} \cosh \left(y(\xi)-y\left(\xi^{\prime}\right)\right) \\
& \times\left[H_{\xi}+\left(U^{2}+2 U_{x} V_{x}-V_{x}^{2}\right) y_{\xi}\right]\left(\xi^{\prime}\right) d \xi^{\prime} \\
& +\frac{1}{4} \int_{0}^{1} e^{-\left|y(\xi)-y\left(\xi^{\prime}\right)\right|} \\
& \times\left[H_{\xi}+\left(U^{2}+2 U_{x} V_{x}-V_{x}^{2}\right) y_{\xi}\right]\left(\xi^{\prime}\right) d \xi^{\prime}, \\
& B_{x}(\xi)=\frac{1}{2(e-1)} \\
& \times \int_{0}^{1} \sinh \left(y(\xi)-y\left(\xi^{\prime}\right)\right) \\
& \times\left[H_{\xi}+\left(U^{2}+2 U_{x} V_{x}-V_{x}^{2}\right) y_{\xi}\right]\left(\xi^{\prime}\right) d \xi^{\prime} \\
& -\frac{1}{4} \int_{0}^{1} \operatorname{sgn}\left(\xi-\xi^{\prime}\right) e^{-\left|y(\xi)-y\left(\xi^{\prime}\right)\right|} \\
& \times\left[H_{\xi}+\left(U^{2}+2 U_{x} V_{x}-V_{x}^{2}\right) y_{\xi}\right]\left(\xi^{\prime}\right) d \xi^{\prime}, \\
& C(\xi)=\frac{1}{e-1} \int_{0}^{1} \cosh \left(y(\xi)-y\left(\xi^{\prime}\right)\right)\left[\left(V U_{x}\right) y_{\xi}\right]\left(\xi^{\prime}\right) d \xi^{\prime} \\
& +\frac{1}{2} \int_{0}^{1} e^{-\left|y(\xi)-y\left(\xi^{\prime}\right)\right|}\left[\left(V U_{x}\right) y_{\xi}\right]\left(\xi^{\prime}\right) d \xi^{\prime}, \\
& C_{x}(\xi)=\frac{1}{e-1} \int_{0}^{1} \sinh \left(y(\xi)-y\left(\xi^{\prime}\right)\right)\left[\left(V U_{x}\right) y_{\xi}\right]\left(\xi^{\prime}\right) d \xi^{\prime} \\
& -\frac{1}{2} \int_{0}^{1} \operatorname{sgn}\left(\xi-\xi^{\prime}\right) e^{-\left|y(\xi)-y\left(\xi^{\prime}\right)\right|} \\
& \times\left[\left(V U_{x}\right) y_{\xi}\right]\left(\xi^{\prime}\right) d \xi^{\prime}, \\
& D(\xi)=\frac{1}{2(e-1)} \\
& \times \int_{0}^{1} \cosh \left(y(\xi)-y\left(\xi^{\prime}\right)\right) \\
& \times\left[H_{\xi}+\left(V^{2}+2 U_{x} V_{x}-U_{x}^{2}\right) y_{\xi}\right]\left(\xi^{\prime}\right) d \xi^{\prime}
\end{aligned}
$$

$$
\begin{aligned}
+ & \frac{1}{4} \int_{0}^{1} e^{-\left|y(\xi)-y\left(\xi^{\prime}\right)\right|} \\
& \times\left[H_{\xi}+\left(V^{2}+2 U_{x} V_{x}-U_{x}^{2}\right) y_{\xi}\right]\left(\xi^{\prime}\right) d \xi^{\prime}, \\
D_{x}(\xi)= & \frac{1}{2(e-1)} \\
& \times \int_{0}^{1} \sinh \left(y(\xi)-y\left(\xi^{\prime}\right)\right) \\
& \quad \times\left[H_{\xi}+\left(V^{2}+2 U_{x} V_{x}-U_{x}^{2}\right) y_{\xi}\right]\left(\xi^{\prime}\right) d \xi^{\prime} \\
& -\frac{1}{4} \int_{0}^{1} \operatorname{sgn}\left(\xi-\xi^{\prime}\right) e^{-\left|y(\xi)-y\left(\xi^{\prime}\right)\right|} \\
& \quad \times\left[H_{\xi}+\left(V^{2}+2 U_{x} V_{x}-U_{x}^{2}\right) y_{\xi}\right]\left(\xi^{\prime}\right) d \xi^{\prime} .
\end{aligned}
$$

From the definition of the characteristic, the evolution equations for $U$ and $V$ in the new variables $(t, \xi)$ take the form

$$
\begin{aligned}
& U_{t}(t, \xi)=u_{t}(t, y)+u_{x}(t, y) y_{t}(t, \xi)=\left(-A-B_{x}\right) \circ y(t, \xi) \\
& V_{t}(t, \xi)=v_{t}(t, y)+v_{x}(t, y) y_{t}(t, \xi)=\left(-C-D_{x}\right) \circ y(t, \xi)
\end{aligned}
$$

With these new variables, we now derive an equivalent system of (13), namely,

$$
\begin{gathered}
y_{t}=U+V, \\
U_{t}=-A-B_{x}, \\
V_{t}=-C-D_{x}, \\
H_{t}=\left[U^{3}-2 U B+V^{3}-2 V D\right]_{0}^{\xi},
\end{gathered}
$$

with $A, A_{x}, B, B_{x}, C, C_{x}, D$, and $D_{x}$ given in (26). Differentiating (29) with respect to $\xi$ yields

$$
\begin{aligned}
y_{\xi t} & =U_{\xi}+V_{\xi}, \\
U_{\xi t} & =\frac{1}{2} H_{\xi}+\left(\frac{1}{2} U^{2}+U_{x} V_{x}-V_{x}^{2}-B-A_{x}\right) y_{\xi}, \\
V_{\xi t} & =\frac{1}{2} H_{\xi}+\left(\frac{1}{2} V^{2}+U_{x} V_{x}-U_{x}^{2}-D-C_{x}\right) y_{\xi}, \\
H_{\xi t} & =\left(3 U^{2}-2 B\right) U_{\xi}-2 U B_{x} y_{\xi}+\left(3 V^{2}-2 D\right) V_{\xi}-2 V D_{x} y_{\xi},
\end{aligned}
$$

which is semilinear with respect to the variables $y_{\xi}, U_{\xi}, V_{\xi}$, and $H_{\xi}$. 


\section{Global Solutions of the Lagrangian Equivalent System}

We consider the Cauchy problem for (29) in the following form:

$$
\begin{gathered}
\varsigma_{t}=U+V, \\
U_{t}=-A-B_{x}, \\
V_{t}=-C-D_{x}, \\
\sigma_{t}=\left[U^{3}-2 U B+V^{3}-2 V D\right]_{0}^{\xi}, \\
h_{t}=0,
\end{gathered}
$$

in the Banach space $E$ given by

$$
E=H_{\mathrm{per}}^{1} \times H_{\mathrm{per}}^{1} \times H_{\mathrm{per}}^{1} \times H_{\mathrm{per}}^{1} \times R
$$

where

$$
H_{\mathrm{per}}^{1}=\left\{f \in H_{\mathrm{loc}}^{1}(R) \mid f(\xi+1)=f(\xi) \forall \xi \in R\right\}
$$

with the norm $\|f\|_{H_{\mathrm{per}}^{1}}=\|f\|_{H^{1}}$ (here we denote $H^{1}([0,1])$ by $H^{1}$ for simplification and the same for other norms in the following), $\varsigma=y-I, \sigma=H-h I$, and $h=H(t, 1)-H(t, 0)$ ( $I$ denotes identity mapping; i.e., $I(\xi)=\xi$ ). Note that there is a bijection $(\varsigma, U, V, \sigma, h) \rightarrow(y, U, V, H)$ between $E$ and $W_{1} \times H_{\text {per }}^{1} \times H_{\text {per }}^{1} \times W$. The advantage of the modification in (31) is that $E$ is a Banach space, which is important for the contraction argument, while $W_{1} \times H_{\text {per }}^{1} \times H_{\text {per }}^{1} \times W$ is not Banach space. In the following, we use the Banach space $E$ and the set of variables $(\varsigma, U, V, \sigma, h)$.

Lemma 1. For any given $X=(\varsigma, U, V, \sigma, h) \in E$, one has that $A, A_{x}, B, B_{x}, C, C_{x}, D$, and $D_{x}$ defined by (26) are locally Lipschitz continuous from $E$ to $H_{p e r}^{1}$. Moreover, one has

$$
\begin{gathered}
A_{x \xi}=\left(A-U V_{x}\right)\left(1+\varsigma_{\xi}\right), \quad A_{\xi}=A_{x}\left(1+\varsigma_{\xi}\right), \\
B_{x \xi}=-\frac{1}{2}\left(\sigma_{\xi}+h\right)+\left(B-\frac{1}{2} U^{2}-U_{x} V_{x}+V_{x}^{2}\right)\left(1+\varsigma_{\xi}\right), \\
B_{\xi}=B_{x}\left(1+\varsigma_{\xi}\right), \\
C_{x \xi}=\left(C-V U_{x}\right)\left(1+\varsigma_{\xi}\right), \\
C_{\xi}=C_{x}\left(1+\varsigma_{\xi}\right), \\
D_{x \xi}=-\frac{1}{2}\left(\sigma_{\xi}+h\right)+\left(D-\frac{1}{2} V^{2}-U_{x} V_{x}+U_{x}^{2}\right)\left(1+\varsigma_{\xi}\right), \\
D_{\xi}=D_{x}\left(1+\varsigma_{\xi}\right) .
\end{gathered}
$$

Proof. We firstly prove that $B_{x}$ given in (26) is locally Lipschitz continuous from $E$ to $H_{\text {per }}^{1}$, and the others follow in the same way. Let $X=(\varsigma, U, V, \sigma, h)$ and $\widetilde{X}=(\widetilde{\varsigma}, \widetilde{U}, \widetilde{V}, \widetilde{\sigma}, \widetilde{h})$ be two elements of $B_{M}$ where $B_{M}=\left\{X=(\varsigma, U, V, \sigma, h)\|\| X \|_{E} \leq\right.$ $M$ \}. We have

$$
\|y\|_{L^{\infty}}=\|I+\varsigma\|_{L^{\infty}} \leq 1+C\|\varsigma\|_{H^{1}} \leq 1+C M
$$

and $\|\tilde{y}\|_{L^{\infty}} \leq 1+C M$. Since $\cosh x$ is locally Lipschitz on $\{x \in R|| x \mid \leq 2(1+C M)\}$, we have

$$
\begin{aligned}
& \left|\cosh \left(y(\xi)-y\left(\xi^{\prime}\right)\right)-\cosh \left(\tilde{y}(\xi)-\tilde{y}\left(\xi^{\prime}\right)\right)\right| \\
& \quad \leq C\left|y(\xi)-\tilde{y}(\xi)-y\left(\xi^{\prime}\right)+\tilde{y}\left(\xi^{\prime}\right)\right| \leq C\|\varsigma-\widetilde{\varsigma}\|_{L^{\infty}},
\end{aligned}
$$

where $C$ is a generic constant depending only on $M$, for all $\xi, \xi^{\prime} \in[0,1]$. It follows that

$$
\begin{aligned}
& \left\|\cosh (y(\xi)-y(\cdot)) U^{2} y_{\xi}(\cdot)-\cosh (\tilde{y}(\xi)-\tilde{y}(\cdot)) \widetilde{U}^{2} \tilde{y}_{\xi}(\cdot)\right\|_{L^{2}} \\
& \leq C\left(\|\varsigma-\widetilde{\varsigma}\|_{L^{\infty}}+\|U-\widetilde{U}\|_{L^{\infty}}+\left\|\varsigma_{\xi}-\widetilde{\varsigma}_{\xi}\right\|_{L^{2}}\right)
\end{aligned}
$$

where we have used the fact that $\left\|U^{2}-\widetilde{U}^{2}\right\|_{L^{\infty}} \leq C\|U-\widetilde{U}\|_{L^{\infty}}$ in $B_{M}$. We handle the other terms of $B$ in the same way and

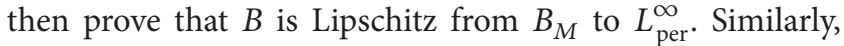
one proves that $B_{x}$ is Lipschitz from $B_{M}$ to $L_{\text {per }}^{\infty}$. Direct computation by the chain rule gives us the expressions (34) for the derivatives of $A, A_{x}, B, B_{x}, C, C_{x}, D$, and $D_{x}$. Using the derivative $B_{x \xi}$ of $B_{x}$, we have

$$
\begin{aligned}
\| B_{x \xi}- & \widetilde{B}_{x \xi} \|_{L^{2}} \\
\leq & C\left(\|B-\widetilde{B}\|_{L^{\infty}}+\|U-\widetilde{U}\|_{L^{\infty}}\right. \\
& +\left\|U_{x}-\widetilde{U}_{x}\right\|_{L^{2}}+\left\|V_{x}-\widetilde{V}_{x}\right\|_{L^{2}} \\
& \left.+\left\|\varsigma_{\xi}-\widetilde{\varsigma}_{\xi}\right\|_{L^{2}}+\left\|\sigma_{\xi}-\widetilde{\sigma}_{\xi}\right\|_{L^{2}}+|h-\widetilde{h}|\right) \\
\leq \| X & -\widetilde{X} \|_{E} .
\end{aligned}
$$

Thus we have proved that $B_{x}$ given in (26) is Lipschitz from $B_{M}$ to $H_{\text {per }}^{1}$. Similarly, $A, A_{x}, B, C, C_{x}, D$, and $D_{x}$ defined in (26) are locally Lipschitz continuous from $B_{M}$ to $H_{\text {per}}^{1}$.

Theorem 2. Let any initial data $\bar{X}=(\bar{\varsigma}, \bar{U}, \bar{V}, \bar{\sigma}, \bar{h}) \in E$ be given. The system (31) admits a unique local solution defined on some time interval $[0, T]$, where $T$ depends only on $\|\bar{X}\|_{E}$.

Proof. To establish the local existence of solutions, one proceeds as in Lemma 1 and obtain that $F(X)$ given by

$$
\begin{aligned}
F(X)=\left(U+V,-A-B_{x},-C-D_{x},\right. \\
\\
\left.\quad\left[U^{3}-2 U B+V^{3}-2 V D\right]_{0}^{\xi}, 0\right),
\end{aligned}
$$

with $X=(\varsigma, U, V, \sigma, h)$, is Lipchitz continuous on any bounded set of $E$. The solutions of system (31) then can be rewritten as

$$
X(t)=\bar{X}+\int_{0}^{t} F(X(\tau)) d \tau
$$


Since $E$ is a Banach space, the theorem then follows from the standard contraction argument.

It remains to show that the local solution can be extended globally in time. Note that global solutions of (31) may not exist for all initial data in $E$. However, they exist when the initial data $\bar{X}=(\bar{\varsigma}, \bar{U}, \bar{V}, \bar{\sigma}, \bar{h})$ belongs to the set $\Gamma$ which is defined as follows.

Definition 3. The set $\Gamma$ is composed of all $(\varsigma, U, V, \sigma, h) \in E$ such that

$$
\text { (i) }(\varsigma, U, V, \sigma, h) \in\left[W^{1, \infty}(R)\right]^{4} \times R \text {, }
$$

(ii) $y_{\xi} \geq 0, H_{\xi} \geq 0, y_{\xi}+H_{\xi}>0$ almost everywhere,

(iii) $y_{\xi} H_{\xi}=y_{\xi}^{2} U^{2}+U_{\xi}^{2}+y_{\xi}^{2} V^{2}+V_{\xi}^{2}$ almost everywhere,

with $y(\xi)=\varsigma(\xi)+\xi, H=\sigma+h I$, where $W_{\text {per }}^{1, \infty}=\left\{f \in W_{\text {loc }}^{1, \infty} \mid\right.$ $f(\xi+1)=f(\xi)$ for all $\xi \in R\}$, which is a Banach space with the norm $\|f\|_{W_{\text {per }}^{1, \infty}}=\|f\|_{W^{1, \infty}}$.

Theorem 2 gives us the existence of local solutions of (31) for initial data in $E$. In the following, we will only consider initial data that belongs to $E \cap\left[W_{\text {per }}^{1, \infty}\right]^{4} \times R$. To obtain that the solution of (31) belongs to $E \cap\left[W_{\text {per }}^{1, \infty}\right]^{4} \times R$, we have to specify the initial condition for (30). Define

$$
\begin{gathered}
\Omega=\left\{\xi \in R|| \bar{\varsigma}_{\xi}(\xi)\left|\leq\left\|\bar{\varsigma}_{\xi}\right\|_{L^{\infty}},\right| \bar{U}_{\xi}(\xi) \mid \leq\left\|\bar{U}_{\xi}\right\|_{L^{\infty}},\right. \\
\left.\left|\bar{V}_{\xi}(\xi)\right| \leq\left\|\bar{V}_{\xi}\right\|_{L^{\infty}},\left|\bar{\sigma}_{\xi}(\xi)\right| \leq\left\|\bar{\sigma}_{\xi}\right\|_{L^{\infty}}\right\} .
\end{gathered}
$$

It is clear that meas $\left(\Omega^{c}\right)=0$. We take $\left(\varsigma_{\xi}, U_{\xi}, V_{\xi}, \sigma_{\xi}\right)(0, \xi)=$ $\left(\bar{\varsigma}_{\xi}, \bar{U}_{\xi}, \bar{V}_{\xi}, \bar{\sigma}_{\xi}\right)(\xi)$ for $\xi \in \Omega$, while $\left(\varsigma_{\xi}, U_{\xi}, V_{\xi}, \sigma_{\xi}\right)(0, \xi)=$ $(0,0,0,0)$ for $\xi \in \Omega^{c}$.

The global existence of the solution for initial data in $\Gamma$ relies essentially on the fact that the set $\Gamma$ is preserved by the flow as the next lemma shows.

Lemma 4. For initial data $\bar{X}=(\bar{\varsigma}, \bar{U}, \bar{V}, \bar{\sigma}, \bar{h}) \in \Gamma$ and some $T>0$, one considers the local solution $X(t)=$ $(\varsigma, U, V, \sigma, h)(t) \in C([0, T], E)$ of system (31). Then

(i) $X(t) \in \Gamma$ for all $t \in[0, T]$,

(ii) $y_{\xi}(t, \xi)>0$ for almost every $t \in[0, T]$ and almost every $\xi \in R$.

Proof. (i) Let $\Omega$ be defined as before. For any given initial data $\bar{X} \in E \cap\left[W_{\text {per }}^{1, \infty}\right]^{4} \times R$, we have that the local solution $X(t) \epsilon$ $C([0, T], E)$ of (31) belongs to $\left[W_{\text {per }}^{1, \infty}\right]^{4} \times R$, which satisfies (41a) for all $t \in[0, T]$. We now show that (41c) holds for any $\xi \in \Omega$ and therefore almost everywhere. Consider a fixed $\xi \in \Omega$ and drop it in the notation. On the one hand, it follows from (30) that

$$
\begin{aligned}
\left(y_{\xi} H_{\xi}\right)_{t}= & y_{\xi t} H_{\xi}+y_{\xi} H_{\xi t} \\
= & \left(U_{\xi}+V_{\xi}\right) H_{\xi}+y_{\xi} \\
& \times\left[\left(3 U^{2}-2 B\right) U_{\xi}+\left(3 V^{2}-2 D\right) V_{\xi}\right. \\
& \left.\quad-2 U B_{x} y_{\xi}-2 V D_{x} y_{\xi}\right] \\
= & U_{\xi} H_{\xi}+V_{\xi} H_{\xi}+3 U^{2} U_{\xi} y_{\xi} \\
& -2 B U_{\xi} y_{\xi}+3 V^{2} V_{\xi} y_{\xi}-2 D V_{\xi} y_{\xi} \\
& -2 U B_{x} y_{\xi}^{2}-2 V D_{x} y_{\xi}^{2},
\end{aligned}
$$

and on the other hand,

$$
\begin{aligned}
\left(y_{\xi}^{2} U^{2}+\right. & \left.U_{\xi}^{2}+y_{\xi}^{2} V^{2}+V_{\xi}^{2}\right)_{t} \\
= & 2 y_{\xi} y_{\xi t} U^{2}+2 y_{\xi}^{2} U U_{t}+2 U_{\xi} U_{\xi t} \\
& +2 y_{\xi} y_{\xi t} V^{2}+2 y_{\xi}^{2} V V_{t}+2 V_{\xi} V_{\xi t} \\
= & U_{\xi} H_{\xi}+V_{\xi} H_{\xi}+3 U^{2} U_{\xi} y_{\xi}+3 V^{2} V_{\xi} y_{\xi} \\
& -2 B U_{\xi} y_{\xi}-2 D V_{\xi} y_{\xi}-2 U B_{x} y_{\xi}^{2}-2 V D_{x} y_{\xi}^{2} .
\end{aligned}
$$

Thus $\left(y_{\xi} H_{\xi}\right)_{t}=\left(y_{\xi}^{2} U^{2}+U_{\xi}^{2}+y_{\xi}^{2} V^{2}+V_{\xi}^{2}\right)_{t}$. Notice that $y_{\xi} H_{\xi}(0)=\left(y_{\xi}^{2} U^{2}+U_{\xi}^{2}+y_{\xi}^{2} V^{2}+V_{\xi}^{2}\right)(0)$, and then $y_{\xi} H_{\xi}(t)=$ $\left(y_{\xi}^{2} U^{2}+U_{\xi}^{2}+y_{\xi}^{2} V^{2}+V_{\xi}^{2}\right)(t)$ for all $t \in[0, T]$ and (41c) has been proved. It remains to prove that the inequalities in (4lb) hold. Define $t^{*}=\sup \left\{t \in[0, T] \mid y_{\xi}\left(t^{\prime}\right) \geq 0\right.$ for all $t^{\prime} \in$ $[0, t]\}$. Assume that $t^{*}<T$. Since $y_{\xi}(t)$ is continuous with respect to $t$, we have $y_{\xi}\left(t^{*}\right)=0$. It follows from (41c) that $U_{\xi}\left(t^{*}\right)=V_{\xi}\left(t^{*}\right)=0$. Furthermore, (30) implies $y_{\xi t}\left(t^{*}\right)=$ $U_{\xi}\left(t^{*}\right)+V_{\xi}\left(t^{*}\right)=0$ and $y_{\xi t t}\left(t^{*}\right)=\left(U_{\xi t}+V_{\xi t}\right)\left(t^{*}\right)=H_{\xi t}\left(t^{*}\right)$. If $H_{\xi t}\left(t^{*}\right)=0$, then $\left(y_{\xi}, U_{\xi}, V_{\xi}, H_{\xi}\right)\left(t^{*}\right)=(0,0,0,0)$ which implies $\left(y_{\xi}, U_{\xi}, V_{\xi}, H_{\xi}\right)(t)=0$ for all $t \in[0, T]$ by the uniqueness of the solution of system (30). This contradicts the fact that $y_{\xi}(0)+H_{\xi}(0)>0$ for all $\xi \in \Omega$. If $H_{\xi t}\left(t^{*}\right)<0$, then $y_{\xi t t}\left(t^{*}\right)<0$. Since $y_{\xi}\left(t^{*}\right)=y_{\xi t}\left(t^{*}\right)=0$, there exists a neighborhood $\omega$ of $t^{*}$ such that $y_{\xi}(t)<0$ for all $t \in \omega /\left\{t^{*}\right\}$. This contradicts the definition of $t^{*}$. Hence, $H_{\xi t}\left(t^{*}\right)>0$. We now have $y_{\xi t t}\left(t^{*}\right)>0$, which conversely implies $y_{\xi}(t)>0$ for all $t \in \omega /\left\{t^{*}\right\}$, which contradicts the fact that $t^{*}<T$. Thus we have proved $y_{\xi}(t) \geq 0$ for all $t \in[0, T]$. We now prove that $H_{\xi} \geq 0$ for all $t \in[0, T]$. This follows from (41c) when $y_{\xi}(t)>0$. If $y_{\xi}(t)=0$, then $U_{\xi}(t)=V_{\xi}(t)=0$ from (41c). As we have seen, $H_{\xi}<0$ would imply that $y_{\xi}\left(t^{\prime}\right)<0$ for some $t^{\prime}$ in a punctured neighborhood of $t$, which is impossible. Hence, $H_{\xi} \geq 0$ for all $t \in[0, T]$. Now we have that $y_{\xi}(t)+$ $H_{\xi}(t) \geq 0$ for all $t \in[0, T]$. If $y_{\xi}\left(t^{\prime}\right)+H_{\xi}\left(t^{\prime}\right)=0$ for some $t^{\prime}$, it then follows that $\left(y_{\xi}, U_{\xi}, V_{\xi}, H_{\xi}\right)\left(t^{\prime}\right)=0$ which implies $\left(y_{\xi}, U_{\xi}, V_{\xi}, H_{\xi}\right)(t)=0$ for all $t \in[0, T]$, which contradicts the fact that $y_{\xi}(0)+H_{\xi}(0)>0$ for all $\xi \in \Omega$. Thus $y_{\xi}(t)+H_{\xi}(t)>0$. 
(ii) Define the set $\Theta=\left\{(t, \xi) \in[0, T] \times R \mid y_{\xi}(t, \xi)=0\right\}$. It follows from Fubini's theorem that

$$
\operatorname{meas}(\Theta)=\int_{R} \operatorname{meas}\left(\Theta_{\xi}\right) d \xi=\int_{[0, T]} \operatorname{meas}\left(\Theta_{t}\right) d t,
$$

where $\Theta_{\xi}=\left\{t \in[0, T] \mid y_{\xi}(t, \xi)=0\right\}$ and $\Theta_{t}=\{\xi \in$ $\left.R \mid y_{\xi}(t, \xi)=0\right\}$. From the above proof, we know that, for all $\xi \in \Omega, \Theta_{\xi}$ consists of isolated points that are countable. This means that meas $\left(\Theta_{\xi}\right)=0$. It follows from (45) and since $\operatorname{meas}\left(\Omega^{c}\right)=0$ that

$$
\text { meas }\left(\Theta_{t}\right)=0 \text { for almost every } t \in[0, T] \text {. }
$$

This implies $y_{\xi}(t, \xi)>0$ for almost all $t$ and therefore $y(t, \xi)$ is strictly increasing and invertible w. r. t. $\xi$.

Theorem 5. For any initial data $\bar{X}=(\bar{y}, \bar{U}, \bar{V}, \bar{\sigma}, \bar{h}) \in$ $\Gamma$, system (31) admits a unique global solution $X(t)=$ $(y, U, V, \sigma, h)(t) \in C^{1}\left(R^{+}, E\right)$. Furthermore, for all $t \geq 0$, one has $X(t) \in \Gamma$, which constructs a continuous semigroup.

Proof. To ensure that the local solution $X=(\varsigma, U, V, \sigma, h) \in$ $C([0, T], E)$ of system (31) can be extended to a global solution, it suffices to show that

$$
\sup _{t \in[0, T)}\|\varsigma(t, \cdot), U(t, \cdot), V(t, \cdot), \sigma(t, \cdot), h(t)\|_{E}<\infty .
$$

We now consider a fixed $t \in[0, T)$ and drop it for simplification. We have

$$
\|H\|_{L^{\infty}} \leq H(1)=H(1)-H(0)=h,
$$

where we have used the fact that $H(0)=0$ and $H$ is an increasing function as $H_{\xi} \geq 0$. It is clear that $h(t)=\bar{h}$ for all $t$. Since $\sigma=H-h I$, we have $\|\sigma\|_{L^{\infty}} \leq 2 h$ and therefore $\sup _{t \in[0, T)}\|\sigma(t, \cdot)\|_{L^{\infty}(R)} \leq 2 \bar{h}$. For $\xi, \xi^{\prime} \in[0,1]$, we have $\left|y(\xi)-y\left(\xi^{\prime}\right)\right| \leq y(1)-y(0)=1$ as $y$ is increasing. Since $H_{\xi}=\left(U^{2}+U_{x}^{2}+V^{2}+V_{x}^{2}\right) y_{\xi}$, we have

$$
\left|\left(U^{2}+2 U_{x} V_{x}-V_{x}^{2}\right) y_{\xi}\right| \leq\left(U^{2}+U_{x}^{2}+V_{x}^{2}+V_{x}^{2}\right) y_{\xi} \leq 2 H_{\xi},
$$

which implies that

$$
\begin{aligned}
\left|B_{x}\right| \leq & \frac{1}{2(e-1)}\left|\int_{0}^{1} \sinh \left(y(\xi)-y\left(\xi^{\prime}\right)\right) 3 H_{\xi}\left(\xi^{\prime}\right) d \xi^{\prime}\right| \\
& +\frac{1}{4}\left|\int_{0}^{1} e^{-\left|y(\xi)-y\left(\xi^{\prime}\right)\right|} 3 H_{\xi}\left(\xi^{\prime}\right) d \xi^{\prime}\right| \\
\leq & C(H(1)-H(0))=C h=C \bar{h},
\end{aligned}
$$

for some constant $C$ and then $\sup _{t \in[0, T)}\left\|B_{x}(t, \cdot)\right\|_{L^{\infty}(R)}<\infty$. Similarly, one can prove that the bounds hold for $A, A_{x}, B, C$, $C_{x}, D$, and $D_{x}$. Since $U_{t}=-A-B_{x}$ and $V_{t}=-C-D_{x}$, we get

$$
\sup _{t \in[0, T)}\|U(t, \cdot)\|_{L^{\infty}(R)}<\infty, \quad \sup _{t \in[0, T)}\|V(t, \cdot)\|_{L^{\infty}(R)}<\infty,
$$

and therefore $\sup _{t \in[0, T)}\|\varsigma(t, \cdot)\|_{L^{\infty}(R)}<\infty$ as $\varsigma_{t}=U+V$. Let

$$
\begin{aligned}
Z(t)= & \|U(t, \cdot)\|_{L^{2}}+\left\|U_{\xi}(t, \cdot)\right\|_{L^{2}}+\|V(t, \cdot)\|_{L^{2}} \\
& +\left\|V_{\xi}(t, \cdot)\right\|_{L^{2}}+\left\|\varsigma_{\xi}(t, \cdot)\right\|_{L^{2}}+\left\|H_{\xi}(t, \cdot)\right\|_{L^{2}} .
\end{aligned}
$$

After taking the $L^{2}$-norms on both sides of (29) and (30), we have

$$
Z(t) \leq Z(0)+C \int_{0}^{t} Z(\tau) d \tau
$$

Gronwall's lemma implies that $\sup _{t \in[0, T)} Z(t)<\infty$, and therefore (47) holds as $\varsigma_{\xi}=y_{\xi}-I$ and $\sigma=H-h I$. It then follows that the map $S_{t}: \Gamma \rightarrow \Gamma \times R^{+}$given by $S_{t}(\bar{X})=$ $X(t)$ generates a continuous semigroup from standard ODE theory.

\section{Global Conservative Solutions of the Original System}

In this section, we show that the global solution of the equivalent system (31) yields a global conservative solution of the original system (13), which constructs a continuous semigroup.

It suffices to establish the correspondence between the Lagrangian equivalent system and the original system.

We first introduce the subsets $F$ and $F_{\alpha}$ of $\Gamma$ defined by

$$
\begin{aligned}
F & =\left\{X=(y, U, V, H) \in \Gamma \mid \frac{1}{1+h}(y+H) \in G\right\}, \\
F_{\alpha} & =\left\{X=(y, U, V, H) \in \Gamma \mid \frac{1}{1+h}(y+H) \in G_{\alpha}\right\},
\end{aligned}
$$

where $h=H(\xi+1)-H(\xi)=H(1)-H(0)$, and the set of relabeling functions $G$ is given by

$$
\begin{gathered}
G=\left\{f \mid f \in W_{\mathrm{loc}}^{1, \infty}(R), f(\xi+1)=f(\xi)+1 \quad \forall \xi \in R,\right. \\
\left.f-I, f^{-1}-I \in W_{\text {per }}^{1, \infty}\right\} .
\end{gathered}
$$

For any $\alpha>1$, we denote the subsets of $G$ by $G_{\alpha}$ as

$$
G_{\alpha}=\left\{f \in G \mid\|f-I\|_{W_{\mathrm{per}}^{1, \infty}}+\left\|f^{-1}-I\right\|_{W_{\mathrm{per}}^{1, \infty}} \leq \alpha\right\},
$$

with a useful characterization: if $f \in G_{\alpha}(\alpha \geq 0)$, then $1 /(1+$ $\alpha) \leq f_{\xi} \leq 1+\alpha$ almost everywhere. Conversely, if $f \in G$ and there exists $c \geq 1$ such that $1 / c \leq f_{\xi} \leq c$ almost everywhere, then $f \in G_{\alpha}$ for some $\alpha$ depending only on $c$. With this useful characterization of $G_{\alpha}$, it is not hard to prove that the space $F$ is preserved by the governing equation (29).

We denote by $\widetilde{G}$ the product group $G \times R$, where the group operation is given by $\left(f_{1}, \gamma_{1}\right) \cdot\left(f_{2}, \gamma_{2}\right)=\left(f_{2} \circ f_{1}, \gamma_{1}+\gamma_{2}\right)$ for any $\left(f_{1}, \gamma_{1}\right),\left(f_{2}, \gamma_{2}\right) \in \widetilde{G}$. We define the map $\Phi: \widetilde{G} \times F \rightarrow F$ as

$$
\Phi\{(f, \gamma),(y, U, V, H)\}=(y \circ f, U \circ f, V \circ f, H \circ f+\gamma) .
$$


The map $\Phi$ defines a group action of $\widetilde{G}$ on $F$. We then consider the quotient space $F / \widetilde{G}$ of $F$ with respect to the group action. We denote the projection $\Pi: F \rightarrow F / \widetilde{G}$ by $\Pi(X)=[X]$. Let us introduce the subset $\widetilde{F}_{0}$ of $F_{0}$ defined as

$$
\widetilde{F}_{0}=\left\{(y, U, V, H) \in F_{0} \mid \int_{0}^{1} y(\xi) d \xi=0\right\} .
$$

It turns out that there exists a bijection between $\widetilde{F}_{0}$ and $F / \widetilde{G}$. To prove this, we introduce the composition map $K: F \rightarrow$ $\widetilde{F}_{0}$ such as $K(X)=K_{2} \circ\left(K_{1}(X)\right)$, where $K_{1}: F \rightarrow F_{0}$ is given by

$$
K_{1}(X)=X \circ f^{-1}
$$

with $X=(y, U, V, H)$ and $f=(1 /(1+h))(y+H) \in F$, and $K_{2}: F_{0} \rightarrow \widetilde{F}_{0}$ is given by

$$
\begin{aligned}
& K_{2}(y, U, V, H) \\
& \quad=(y(\xi-a), U(\xi-a), V(\xi-a), H(\xi-a)+(1+h) a)
\end{aligned}
$$

with $a=\int_{0}^{1} y(\xi) d \xi$. It is not hard to check that $K_{1}(X) \in F_{0}$, that is, $(1 /(1+\bar{h}))(\bar{y}+\bar{H})=I$, and $K_{2}(y, U, V, H) \in \widetilde{F}_{0}$. Thus $K(X)$ belongs to the same equivalence class as $X$, and there corresponds to $K$ a map $\widetilde{K}: F / \widetilde{G} \rightarrow \widetilde{F}_{0}$ given by $\widetilde{K}([X])=K(X)$, where $[X] \in F / \widetilde{G}$ denotes the equivalence class of $X \in F$. It is not hard to check that $K_{\mid \widetilde{F}_{0}}=I_{\mid \widetilde{F}_{0}}$. Thus, $\widetilde{K} \circ \Pi_{\mid \widetilde{F}_{0}}=I_{\mid \widetilde{F}_{0}}$ and therefore $\widetilde{K}$ is a bijection from $F / \widetilde{G}$ to $\widetilde{F}_{0}$. Note that any topology defined on $\widetilde{F}_{0}$ is naturally transported into $F / \widetilde{G}$ by the bijection $\widetilde{K}$. That is, if we equip $\widetilde{F}_{0}$ with the metric induced by the $E$-norm, that is $d_{\widetilde{F}_{0}}\left(X, X^{\prime}\right)=$ $\left\|X-X^{\prime}\right\|_{E}$ for all $X, X^{\prime} \in \widetilde{F}_{0}$, which is complete, then the topology on $F / \widetilde{G}$ is defined by a complete metric given by $d_{F / \widetilde{G}}\left([X],\left[X^{\prime}\right]\right)=\left\|\widetilde{K}(X)-\widetilde{K}\left(X^{\prime}\right)\right\|_{E}$ for any $[X],\left[X^{\prime}\right] \in F / \widetilde{G}$.

For any initial data $\bar{X} \in F$, we denote by $S: F \times R^{+} \rightarrow F$ the continuous semigroup with the solution $X(t)$ of (29). Equation (13) is invariant with respect to relabeling. That is, for any $X \in F$ and $(f, \gamma) \in \widetilde{G}$, we have that $S_{t}(\Phi\{(f, \gamma), X\})=$ $\Phi\left\{(f, \gamma), S_{t}(X)\right\}$. Therefore, the map $\widetilde{S}_{t}: F / \widetilde{G} \rightarrow F / \widetilde{G}$ given by $\widetilde{S}_{t}([X])=\left[S_{t} X\right]$ is well defined, which generates a continuous semigroup.

To obtain a semigroup of solution for (13), we have to consider the space $\Xi$, which characterizes the solutions in the original system:

$$
\begin{array}{r}
\Xi=\left\{(z, \mu) \mid z \in H_{\text {per }}^{1} \times H_{\text {per }}^{1}\right. \text { and } \\
\left.\mu_{\mathrm{ac}}=\left(u^{2}+u_{x}^{2}+v^{2}+v_{x}^{2}\right) d x\right\},
\end{array}
$$

where $z=(u, v)$ and $\mu$ is a positive periodic Radon measure with $\mu_{\mathrm{ac}}$ being its absolute continuous part.

In the following, we establish a bijection between $F / \widetilde{G}$ and $\Xi$ to transport the continuous semigroup obtained in the Lagrangian equivalent system (functions in $F / \widetilde{G}$ ) into the original system (functions in $\Xi$ ).
Let us first introduce the mapping $M$, which transforms the Lagrangian equivalent system into the original system. In the other direction, we obtain the energy density $\mu$ in the original system, by pushing forward by $y$ the energy density $H_{\xi} d \xi$ in Lagrangian equivalent system, where the pushforward $f_{\#} \nu$ of a measure $\nu$ by a measurable function $f$ is defined as

$$
f_{\#} v(B)=\nu\left(f^{-1}(B)\right)
$$

for all Borel set $B$. Let $(z, \mu)$ be defined as

$$
\begin{gathered}
z(x)=Z(\xi) \text { for any } \xi \text { such that } x=y(\xi), \\
\mu=y_{\#}\left(H_{\xi} d \xi\right)
\end{gathered}
$$

with $z(x)=(u, v)(x)$ and $Z(\xi)=(U, V)(\xi)$. We have that $(z, \mu) \in \Xi$, which does not depend on the representative $X=$ $(y, U, V, H) \in F$ of $[X]$ that we choose. We denote by $M$ : $F / \widetilde{G} \rightarrow \Xi$ the mapping to any $[X] \in F / \widetilde{G}$ and $(z, \mu) \in \Xi$ given by ((63a) and (63b)), which transforms the Lagrangian equivalent system into the original system.

We are led to the mapping $L: \Xi \rightarrow F / \widetilde{G}$, which corresponds to the transformation from the original system into the Lagrangian equivalent system defined as follows.

Definition 6. For any $(z, \mu) \in \Xi$, let

$$
\begin{gathered}
h=\mu([0,1)), \\
y(\xi)=\sup \left\{y \mid F_{\mu}(y)+y<(1+h) \xi\right\}, \\
U(\xi)=u \circ y(\xi), \quad V(\xi)=v \circ y(\xi), \\
H(\xi)=(1+h) \xi-y(\xi),
\end{gathered}
$$

where $z=(u, v)$. We define $L(z, \mu) \in F / \widetilde{G}$ as the equivalence class of $(y, U, V, H)$. Here, the function $F_{\mu}$ associated with any positive periodic Radon measure $\mu$ is given by

$$
F_{\mu}(x)= \begin{cases}\mu([0, x)) & \text { if } x>0 \\ 0 & \text { if } x=0 \\ -\mu([x, 0)) & \text { if } x<0\end{cases}
$$

which is lower-semicontinuous, increasing and moreover, $F_{\mu}(b)-F_{\mu}(a)=\mu([a, b))$ for all $a<b$ in $R$.

Remark 7. From the definition of $y, U, V, H$ in ((64), (65), (66), and (67)), we can check that $X=(y, U, V, H) \in E$ and $X=(y, U, V, H)$ satisfies (41a), (41b), and (41c), respectively. Moreover, by definition (67), we have $(1 /(1+h))(y+H)=I$. Thus $X=(y, U, V, H) \in F_{0}$.

We claim that the transformation from the original system into the Lagrangian equivalent system is a bijection.

Theorem 8. The mappings $M$ and $L$ are invertible; that is,

$$
L \circ M=I_{F / \widetilde{G}}, \quad M \circ L=I_{\Xi} .
$$


Proof. Let $[X] \in F / \widetilde{G}$ be given. We denote by $X=$ $(y, U, V, N, H)=\widetilde{K}([X])$ a representative of $[X]$ and $(z, \mu)$ given by $((63 \mathrm{a})$ and $(63 \mathrm{~b}))$ for this particular $X$. It follows from the definition of $\widetilde{K}$ that $X \in \widetilde{F}_{0}$. Let $\bar{X}=(\bar{y}, \bar{U}, \bar{V}, \bar{H})$ be the representative of $L(z, \mu)$ in $F_{0}$ given by (64), (65), (66), and (67). It suffices to show that $(\bar{y}, \bar{U}, \bar{V}, \bar{H})=(y, U, V, H)$ and therefore $L \circ M=I_{F / \widetilde{G}}$. Set

$$
g(x)=\sup \{\xi \in R \mid y(\xi)<x\} .
$$

Using the fact that $y$ is increasing and continuous, it is not hard to know that

$$
y(g(x))=x
$$

and $y^{-1}([a, b))=[g(a), g(b))$ for any $a<b$ in $R$. It follows from $(63 \mathrm{~b})$ that

$$
\begin{aligned}
\mu([a, b)) & =\int_{y^{-1}([a, b))} H_{\xi} d \xi=\int_{g(a)}^{g(b)} H_{\xi} d \xi \\
& =H(g(b))-H(g(a)) .
\end{aligned}
$$

Since $X \in F_{0}$ and $y+H=(1+h) I$, we have

$$
\begin{aligned}
& H(g(b))=(1+h) g(b)-b, \\
& H(g(a))=(1+h) g(a)-a .
\end{aligned}
$$

From (72) and the definition of $F_{\mu}$, we get

$$
F_{\mu}(x)+x=(1+h)(g(x)-g(0)) .
$$

It then follows from the definition of $\bar{y}$ that

$$
\bar{y}(\xi)=\sup \{x \in R \mid g(x)<\xi+g(0)\} .
$$

For any given $\xi \in R$, using the fact that $y$ is increasing and (71), we have $\bar{y}(\xi) \leq y(\xi+g(0))$. Assume $\bar{y}(\xi)<y(\xi+g(0))$, and there then exists $x$ such that $\bar{y}(\xi)<x<y(\xi+g(0))$ and (75) implies that $g(x) \geq \xi+g(0)$. Therefore, $x \geq y(\xi+g(0))$, which contradicts the fact that $x<y(\xi+g(0))$. Hence, we have

$$
\bar{y}(\xi)=y(\xi+g(0)) .
$$

Since $\bar{X} \in F_{0}, \bar{H}(\xi)=(1+h) \xi-\bar{y}(\xi)$, and $(y+H)(\xi+g(0))=$ $(1+h)(\xi+g(0))$, we have

$$
\bar{H}(\xi)=H(\xi+g(0))-(1+h) g(0) .
$$

It is not hard to know that

$$
\bar{U}(\xi)=U(\xi+g(0)), \quad \bar{V}(\xi)=V(\xi+g(0)) .
$$

It then follows that $L \circ M=I_{F / \widetilde{G}}$.

Given $(z, \mu)$ in $\Xi$, we denote by $(y, U, V, H)$ the representative of $L(z, \mu)$ in $F_{0}$ given by (64), (65), (66), and (67). Then, let $(\bar{z}, \bar{\mu})=M \circ L(z, \mu)$. Let $g$ be defined as before by (70). The same computation that leads to (74) now gives

$$
F_{\bar{\mu}}(x)+x=(1+h)(g(x)-g(0)) .
$$

Given $\xi \in R$, we consider an increasing sequence $x_{i}$ converging to $y(\xi)$ which is guaranteed by (65), such that $F_{\mu}\left(x_{i}\right)+x_{i}<(1+h) \xi$. Let $i$ tend to infinity and since $F_{\mu}$ is lower semicontinuous, we have $F_{\mu}(y(\xi))+y(\xi) \leq(1+h) \xi$. Take $\xi=g(x)$ and then we get

$$
F_{\mu}(x)+x \leq(1+h) g(x) .
$$

By the definition of $g$, there exists an increasing sequence $\xi_{i}$ converging to $g(x)$ such that $y\left(\xi_{i}\right)<x$. It follows from the definition of $y$ in $(65)$ that $F_{\mu}(x)+x \geq(1+h) \xi_{i}$. Passing to the limit, we obtain $F_{\mu}(x)+x \geq(1+h) g(x)$ which, together with (80), yields

$$
F_{\mu}(x)+x=(1+h) g(x) .
$$

We obtain that $\bar{\mu}=\mu$ by comparing (81) and (79). It is clear from the definitions that $\bar{z}=z$. Hence, $(\bar{z}, \bar{\mu})=(z, \mu)$ and $M \circ L=I_{\Xi}$.

Now we have established a bijection between the two equivalent systems, and thus the topology defined in $F / \widetilde{G}$ can be transported into $\Xi$. Let us define the distance $d_{\Xi}$ on $\Xi$ as

$$
d_{\Xi}((z, \mu),(\bar{z}, \bar{\mu}))=d_{F / \widetilde{G}}(L(z, \mu), L(\bar{z}, \bar{\mu})),
$$

which makes the bijection $L$ between $\Xi$ and $F / \widetilde{G}$ into an isometry. Since $F / \widetilde{G}$ equipped with $d_{F / \widetilde{G}}$ is a complete metric space, it is not hard to check that $\Xi$ equipped with the metric $d_{\Xi}$ is also a complete metric space. For each $t \in R$, we define the mapping $T_{t}: \Xi \rightarrow \Xi$ as

$$
T_{t}=M \widetilde{S}_{t} L
$$

Our main results are given as follows.

Theorem 9. Let $(\bar{z}, \bar{\mu}) \in \Xi$ be given. If one denotes by $t \rightarrow$ $(z(t), \mu(t))=T_{t}(\bar{z}, \bar{\mu})$ the corresponding trajectory, $z=(u, v)$ is a weak solution of the modified coupled two-component Camassa-Holm system (13), which constructs a continuous semigroup. Moreover, $\mu$ is a weak solution of the following transport equation for the energy density

$$
\mu_{t}+[(u+v) \mu]_{x}=\left(u^{3}-2 B u+v^{3}-2 D v\right)_{x} .
$$

Furthermore, one has

$$
\mu(t)([0,1))=\mu(0)([0,1)) \quad \forall t,
$$

and for almost all $t$, there holds

$$
\begin{aligned}
\mu(t)([0,1)) & =\mu_{a c}(t)([0,1)) \\
& =\|z(t)\|_{H_{p e r}^{1}}^{2}=\|u(t)\|_{H_{p e r}^{1}}^{2}+\|v(t)\|_{H_{p e r}^{1}}^{2} \\
& =\mu(0)([0,1)) .
\end{aligned}
$$

Thus, the unique solution described here is a conservative weak solution of the system (13). 
Proof. To prove $z=(u, v)$ is a weak solution of the original system (13), it suffices to show that, for all $\phi \in C^{\infty}\left(R^{+} \times R\right)$ with compact support,

$$
\begin{array}{r}
\int_{R^{+} \times R}\left[-u \phi_{t}+(u+v) u_{x} \phi\right](t, x) d x d t \\
=-\int_{R^{+} \times R}\left[\left(A+B_{x}\right) \phi\right](t, x) d x d t, \\
\int_{R^{+} \times R}\left[-v \phi_{t}+(u+v) v_{x} \phi\right](t, x) d x d t \\
=-\int_{R^{+} \times R}\left[\left(C+D_{x}\right) \phi\right](t, x) d x d t,
\end{array}
$$

where $A, B_{x}, C$, and $D_{x}$ are given by (13). Let the solution $(y, U, V, H)(t)$ of (29) be a representative of $L(z(t), \mu(t))$. Since $y(t, \xi)$ is Lipschitz and invertible w. r. t. $\xi$ for almost all $t$, we can use the change of variables $x=y(t, \xi)$ and get

$$
\begin{aligned}
\int_{R^{+} \times R}\left[-u \phi_{t}+(u+v) u_{x} \phi\right](t, x) d x d t \\
=\int_{R^{+} \times R}\left[-\left(U y_{\xi}\right)(t, \xi) \phi_{t}(t, y(t, \xi))+\left((U+V) U_{\xi}\right)\right. \\
\quad \times(t, \xi) \phi(t, y(t, \xi))] d \xi d t .
\end{aligned}
$$

Using the identities $y_{t}=U+V$ and $y_{\xi t}=U_{\xi}+V_{\xi}$, it then follows from (29) that

$$
\begin{gathered}
\int_{R^{+} \times R}\left[-U y_{\xi} \phi_{t}(t, y)+(U+V) U_{\xi} \phi(t, y)\right] d \xi d t \\
=\frac{1}{2} \int_{R^{+} \times R^{2}}\left\{-U V_{x} y_{\xi}+\frac{1}{2} \operatorname{sgn}\left(\xi-\xi^{\prime}\right)\right. \\
\left.\times\left[H_{\xi}+\left(U^{2}+2 U_{x} V_{x}-V_{x}^{2}\right) y_{\xi}\right]\right\}\left(\xi^{\prime}\right) \\
\cdot e^{-\left|y(\xi)-y\left(\xi^{\prime}\right)\right|} \phi(t, y(\xi)) y_{\xi}(\xi) d \xi^{\prime} d \xi d t .
\end{gathered}
$$

On the other hand, using the change of variables $x=y(t, \xi)$ and $x^{\prime}=y\left(t, \xi^{\prime}\right)$ and since $y$ is an increasing function, we obtain that

$$
\begin{aligned}
&-\int_{R^{+} \times R}\left[\left(A+B_{x}\right) \phi\right](t, x) d x d t \\
&=\frac{1}{2} \int_{R^{+} \times R^{2}}[-u v_{x}+\operatorname{sgn}\left(\xi-\xi^{\prime}\right) \\
&\left.\times\left(u^{2}+\frac{1}{2} u_{x}^{2}+u_{x} v_{x}+\frac{1}{2} v^{2}-\frac{1}{2} v_{x}^{2}\right)\right] \\
& \times\left(t, y\left(\xi^{\prime}\right)\right) \\
& \cdot e^{-\left|y(\xi)-y\left(\xi^{\prime}\right)\right|} \phi(t, y(\xi)) y_{\xi}\left(\xi^{\prime}\right) y_{\xi}(\xi) d \xi^{\prime} d \xi d t .
\end{aligned}
$$

It then follows from (41c) that

$$
\begin{aligned}
&-\int_{R^{+} \times R}\left[\left(A+B_{x}\right) \phi\right](t, x) d x d t \\
&=\frac{1}{2} \int_{R^{+} \times R^{2}}\left\{-U V_{x} y_{\xi}+\frac{1}{2} \operatorname{sgn}\left(\xi-\xi^{\prime}\right)\right. \\
& \times {\left.\left[H_{\xi}+\left(U^{2}+2 U_{x} V_{x}-V_{x}^{2}\right) y_{\xi}\right]\right\}\left(\xi^{\prime}\right) } \\
& \cdot e^{-\left|y(\xi)-y\left(\xi^{\prime}\right)\right|} \phi(t, y(\xi)) y_{\xi}(\xi) d \xi^{\prime} d \xi d t .
\end{aligned}
$$

By comparing (89) and (91), we can see that

$$
\begin{aligned}
& \int_{R^{+} \times R} {\left[-U y_{\xi} \phi_{t}(t, y)+(U+V) U_{\xi} \phi(t, y)\right] d \xi d t } \\
&=-\int_{R^{+} \times R}\left[\left(A+B_{x}\right) \phi\right](t, x) d x d t .
\end{aligned}
$$

Hence, the first identity in (87) holds. The second identity in (87) follows in the same way. One can easily check that $\mu(t)$ is solution of (84). It follows from the definitiong in (70) that

$$
y^{-1}([0,1))=[g(0), g(1)), \quad g(x+1)=g(x)+1 .
$$

Since $H_{\xi}$ is periodic, (63b) implies that

$$
\begin{aligned}
\mu(t)([0,1)) & =\int_{[g(0), g(0)+1)} H_{\xi} d \xi \\
& =\int_{[0,1)} H_{\xi} d \xi=H(t, 1)-H(t, 0),
\end{aligned}
$$

which is constant in time from (29). Thus, we have proved (85). Since $y_{\xi}(t, \xi)>0$ almost everywhere for almost every $\xi \in R$, then it follows from (41c) that

$$
\begin{aligned}
\mu(t)(B) & =\int_{y^{-1}(B)} H_{\xi} d \xi \\
& =\int_{y^{-1}(B)}\left(U^{2}+\frac{U_{\xi}^{2}}{y_{\xi}^{2}}+V^{2}+\frac{V_{\xi}^{2}}{y_{\xi}^{2}}\right) y_{\xi} d \xi
\end{aligned}
$$

for any Borel set $B$. Using the fact that $y$ is one-to-one and $u_{x} \circ y y_{\xi}=U_{\xi}, v_{x} \circ y y_{\xi}=V_{\xi}$ almost everywhere, (95) implies that

$$
\mu(t)(B)=\int_{B}\left(u^{2}+u_{x}^{2}+v^{2}+v_{x}^{2}\right)(t, x) d x .
$$

Hence, (86) holds. This completes the proof.

\section{Conclusion}

We consider in this paper the periodic conservative property of the solutions for the modified coupled two-component Camassa-Holm system with peakons. By introducing a skillfully defined characteristic, together with a set of newly 
defined variables, the original system is converted into a Lagrangian equivalent system, from which periodic conservative solution is obtained. It is the transformation together with the associated properties that overcomes the difficulties inherent in the multicomponent system and allows for the continuity of the solution beyond collision time, leading to the desired results.

\section{Acknowledgments}

The authors would like to thank the referees for constructive suggestions and comments. The paper is supported by the Major State Basic Research Development Program 973 (no. 2012CB215202) and the National Natural Science Foundation of China (no. 61134001).

\section{References}

[1] Y. Fu, Y. Liu, and C. Qu, "Well-posedness and blow-up solution for a modified two-component periodic Camassa-Holm system with peakons," Mathematische Annalen, vol. 348, no. 2, pp. 415448,2010

[2] Y. Fu and C. Qu, "Well posedness and blow-up solution for a new coupled Camassa-Holm equations with peakons," Journal of Mathematical Physics, vol. 50, no. 1, Article ID 012906, 25 pages, 2009.

[3] A. Constantin, "The Hamiltonian structure of the CamassaHolm equation," Expositiones Mathematicae, vol. 15, no. 1, pp. 53-85, 1997.

[4] R. Camassa and D. D. Holm, "An integrable shallow water equation with peaked solitons," Physical Review Letters, vol. 71, no. 11, pp. 1661-1664, 1993.

[5] A. Constantin, "On the scattering problem for the CamassaHolm equation," Proceedings of the Royal Society of London A, vol. 457, no. 2008, pp. 953-970, 2001.

[6] A. Constantin and D. Lannes, "The hydrodynamical relevance of the Camassa-Holm and Degasperis-Procesi equations," Archive for Rational Mechanics and Analysis, vol. 192, no. 1, pp. 165-186, 2009.

[7] A. Constantin and J. Escher, "Wave breaking for nonlinear nonlocal shallow water equations," Acta Mathematica, vol. 181, no. 2, pp. 229-243, 1998.

[8] A. Constantin, "Existence of permanent and breaking waves for a shallow water equation: a geometric approach," Annales de l'Institut Fourier, vol. 50, no. 2, pp. 321-362, 2000.

[9] A. Bressan and A. Constantin, "Global conservative solutions of the Camassa-Holm equation," Archive for Rational Mechanics and Analysis, vol. 183, no. 2, pp. 215-239, 2007.

[10] H. Holden and X. Raynaud, "Global conservative solutions of the Camassa-Holm equation-a Lagrangian point of view," Communications in Partial Differential Equations, vol. 32, no. 10-12, pp. 1511-1549, 2007.

[11] H. Holden and X. Raynaud, "Periodic conservative solutions of the Camassa-Holm equation," Annales de l'Institut Fourier, vol. 58, no. 3, pp. 945-988, 2008.

[12] H. Holden and X. Raynaud, "Global conservative multipeakon solutions of the Camassa-Holm equation," Journal of Hyperbolic Differential Equations, vol. 4, no. 1, pp. 39-64, 2007.

[13] A. Bressan and A. Constantin, "Global dissipative solutions of the Camassa-Holm equation," Analysis and Applications, vol. 5, no. 1, pp. 1-27, 2007.
[14] L. Tian and Y. Xu, "Attractor for a viscous coupled CamassaHolm equation," Advances in Difference Equations, vol. 2010, Article ID 512812, 30 pages, 2010.

[15] L. Tian, Y. Wang, and J. Zhou, "Global conservative and dissipative solutions of a coupled Camassa-Holm equations," Journal of Mathematical Physics, vol. 52, no. 6, Article ID 063702, 29 pages, 2011.

[16] Y. Wang and Y. Song, "On the global existence of dissipative solutions for the modified coupled Camassa-Holm system," Soft Computing, vol. 17, no. 1, pp. 2007-2019, 2013.

[17] Z. Shen, Y. Wang, H. Karimi, and Y. Song, "On the multipeakon dissipative behavior of the modified coupled Camassa-Holm model for shallow water system," Mathematical Problems in Engineering, vol. 2013, Article ID 107450, 11 pages, 2013. 


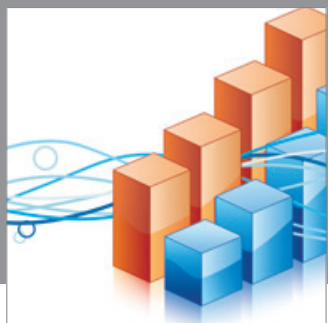

Advances in

Operations Research

mansans

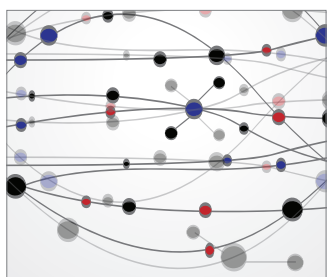

The Scientific World Journal
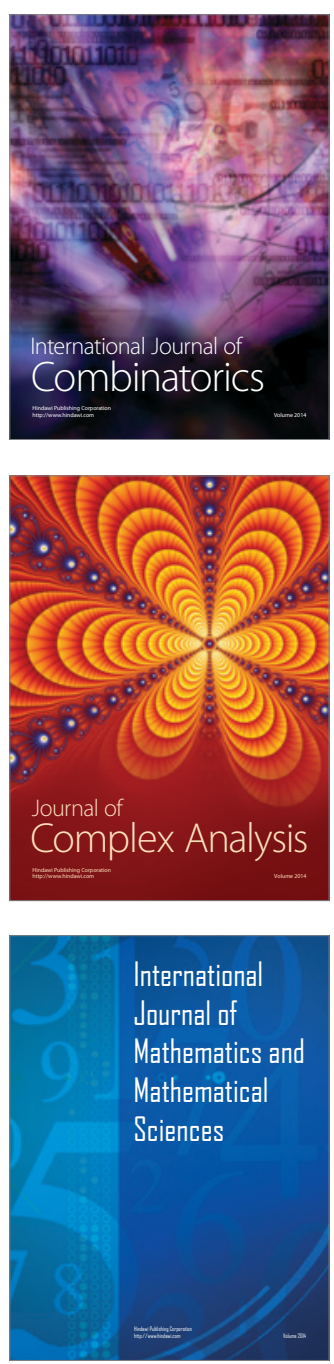
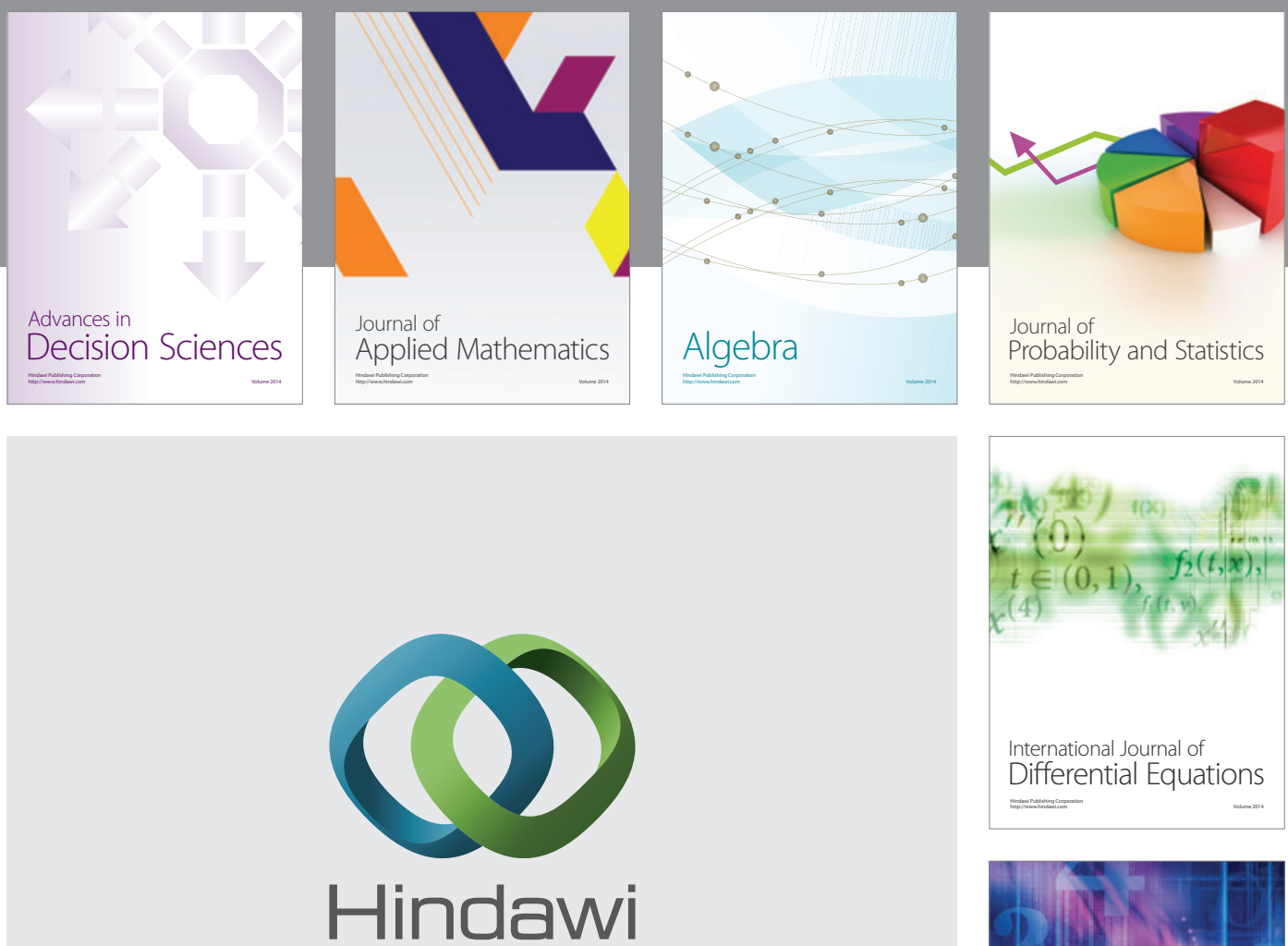

Submit your manuscripts at http://www.hindawi.com
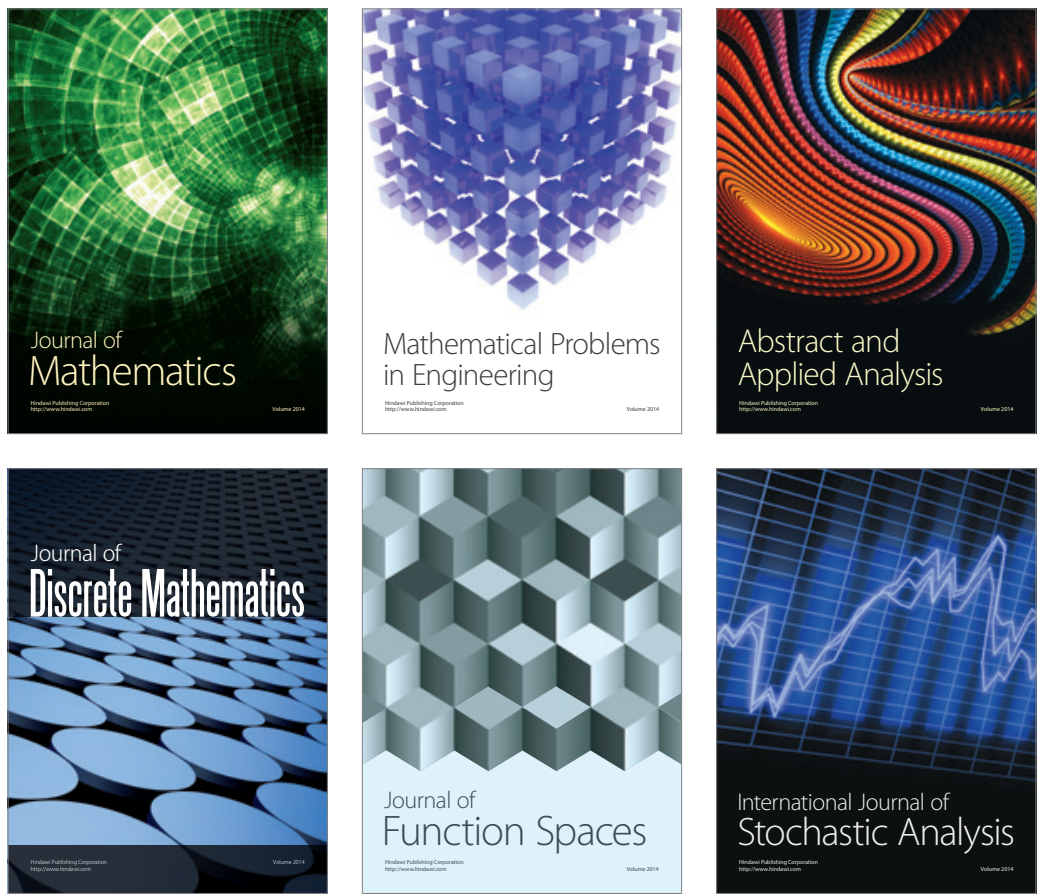

Journal of

Function Spaces

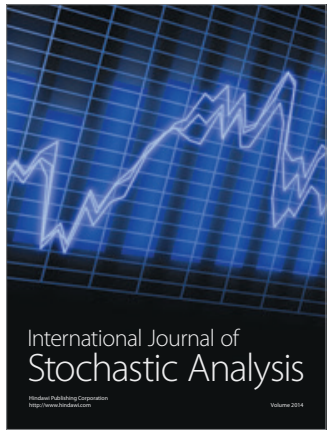

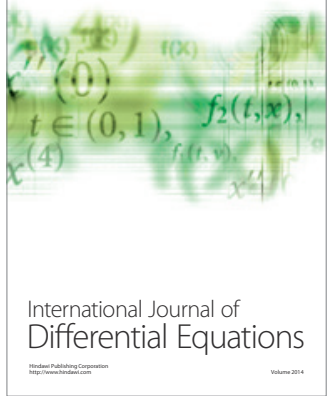
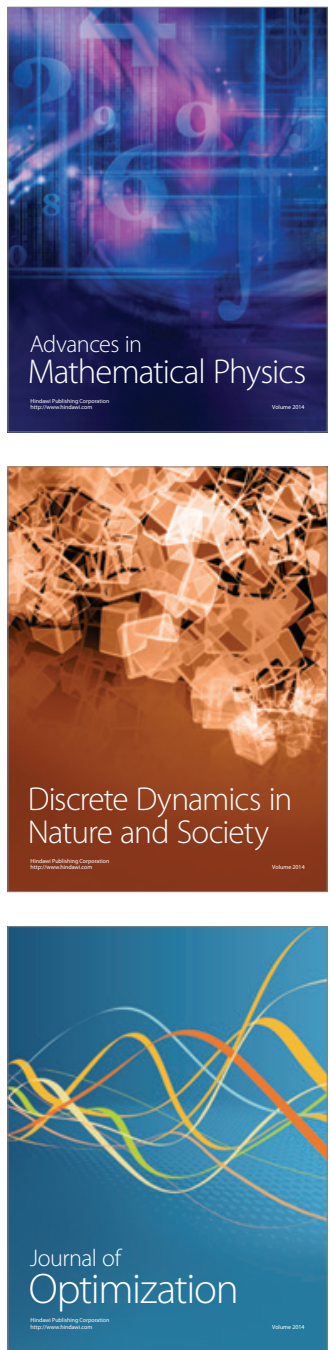